\title{
EFFECTS OF STRUCTURE BOUNDARY CONDITIONS AND SNOW-PACK PROPERTIES ON SNOW-CREEP PRESSURES
}

\author{
by \\ D.M. McClung \\ (Institute for Research in Construction, National Research Council of Canada, 3650 Wesbrook Mall, \\ Vancouver, British Columbia V6S 2L2, Canada) \\ and \\ J.O. Larsen
}

(Norwegian Geotechnical Institute, Postboks 40 Taasen, N-0801 Oslo 8, Norway)

\begin{abstract}
Structures placed in deep snow covers are subject to forces caused by interruption of the down-slope snow-pack deformation components. The resulting creep pressures are often the primary design consideration. In this paper, accurate field data (pressures) and theoretical analysis of the problem using a linear creep law to define snow deformation are presented. Results include analytical expressions for the pressures, and it is demonstrated that the resulting linear theory underestimates the mean pressures by about $20 \%$. Higher accuracy will require that a nonlinear deformation law be formulated.
\end{abstract}

\section{INTRODUCTION}

When structures are erected in deep snow covers, snowcreep pressures are of ten the primary design consideration. Important examples include avalanche defences in starting zones, and ski-lift and power-line towers. Although it is relatively easy to design structures which can withstand creep pressures, the cost penalty for structures which are stronger than necessary is of ten prohibitive. Conversely, the failure of structures buried in deep snow covers can be very expensive and potentially dangerous. These considerations underline the importance of accuracy in the specification of the expected creep loads.

For a given snow-pack, two elements control the distribution and magnitude of forces on structures: (1) the boundary conditions on the face of the structure and at positions where the snow-pack is in contact with the ground, and (2) the rheology of the material. In this paper, the effects of boundary conditions are quantified and compared with field data from a plane-strain configuration. Calculations are given over the range of expected boundary conditions appropriate to the data. In addition, simple depth variations in snow-pack density and stiffness are explored using linear rheology. Taken together, the data and calculations indicate the direction that must be taken for a complete definition of design loads. In particular, we are able to show that in the future the focus of attention must be on non-linear rheology.

\section{MODELLING CONCEPTS}

Alpine snow has a unique combination of physical properties which have not yet been formalized in a non-linear deformation law suitable for engineering applications. These properties include: (1) high porosity; (2) high temperature (relative to its melting point); and (3) very low strength (it is the weakest bonded natural geotechnical material). These properties combine to produce slow deformation which can occur even without an applied load. The high porosity results in continuous densification throughout the winter, through irreversible deformation (mainly from grain re-arrangement). This viscous (or plastic) deformation may be described as non-steady creep.

Given the absence of a non-linear formulation, a linear deformation law is applied in this paper. The linear theory yields analytical expressions for the expected loads on the structure, and it provides information on the importance and character of non-linear effects. Our approach is to generalize linear visco-elastic behaviour from a model which is characterized in one dimension as a four-parameter Burger Fluid (see Flügge, 1975, p. 22). This results in an engineering formulation for describing interruption of slow, viscous creep by a rigid structure on a slope with a deep snow cover. Transient visco-elastic effects induced by loading (new snowfall) are ignored; field data (McClung, 1975) show that transient creep rates in new snow layers persist for several days following a storm.

Even when rapid (transient) creep rates are not present, the creep loads on a structure are still time-dependent because alpine snow is continuously densifying and settling, making steady-state creep impossible. However, the effect is very slow for a deep snow cover which has been present for several months on a slope. For design purposes, the time of greatest interest is late winter or early spring when snow depth is at a maximum and densification is slow. With a linear model it is possible to treat this aspect of time-dependence by exploring slow variations in the moduli as time proceeds but with constant values at a given instant of time.

Generalization of the one-dimensional model to a three-dimensional one (including both deviatoric and hydrostatic components) is well known. Lang and Nakamura (1984) have provided a rigorous treatment. For long-term response (with initial stresses and transients ignored), the linear constitutive equation becomes

$$
\sigma_{i j}=2 \mu \dot{e}_{i j}+\left[\eta-\frac{2}{3} \mu\right] \dot{e}_{k k} \delta_{i j}
$$

where $\mu, n$ are shear and bulk viscosity, and $\sigma_{i j}, \dot{e}_{i j}$ are stress- and strain-rate tensor components (rectangular Cartesian coordinates in the $i, j$ directions), $\delta_{i j}$ is the Kronecker delta. Equation (1) represents a linear, compressible Newtonian viscous fluid neglecting the static pressure term. The static term is not necessary for describing alpine snow (Salm, 1967) because a state of rest is not possible. We believe Equation (1) represents a sufficiently accurate linear representation of alpine snow for engineering applications. For alpine snow deforming slowly, 
strains due to viscous creep will exceed initial elastic strains by several orders of magnitude.

Information about values of bulk viscosity is scarce, but it is natural to relate the shear and bulk viscosity to the viscous Poisson's ratio described by Reiner (1946, 1949):

$$
v=\frac{3 n-2 \mu}{2(3 n+\mu)} .
$$

Values for $v$ have been summarized by Salm (1977) and estimates of $\mu$ have been given by Haefeli (1967). This pair of parameters can be used to describe linear creep deformation in general but, for plane-strain solutions, $v$ will be the only parameter to appear.

Both $\mu$ and $\eta$ may depend on snow density, temperature, structure, and time for a linear theory. For a non-linear formulation, they may also depend on invariants of the stress- and strain-rate tensor (a case not considered in this paper).

\section{SNOW GLIDING}

The boundary conditions at positions where the snow-pack is in contact with the ground are crucial in determining structure forces when the ground is smooth and wet. Snow glide (slip of the entire snow-pack over sloping ground) can be initiated when the interface temperature between the snow-pack and the ground is at $0{ }^{\circ} \mathrm{C}$ and for slope angles in excess of $15^{\circ}$. When vigorous gliding takes place, the highest forces on structures are produced, and therefore no serious model would exclude this force component.

The fundamental problem of snow gliding is to relate the snow-pack drag to the glide velocity. The present theory contains the assumption that glide occurs by creep over the ground-roughness elements. When the interface is at $0^{\circ} \mathrm{C}$, there is a guaranteed presence of free water. This condition implies that the velocity field is tangential to the interface at positions where the snow-pack contacts roughness elements and that there is negligible shear stress at all contact points. At positions for which the snow-pack is not in contact with the interface, the drag is negligible (McClung and Clarke, 1987). Assuming the deformation field is governed by Equation (1), the tangential snow-pack drag $\tau_{0}$ is related to the glide velocity $U$ by

$$
\tau_{0}=\frac{\mu U}{2(1-v) D^{\mathrm{A}}(x, y)} \equiv \frac{\mu U}{D^{*}}
$$

where $D^{\mathrm{A}}(x, y)$, the stagnation depth, is a function of the geometry of the interface ( $x$ and $y$ are up- and cross-slope directions), and $A$ is the area for which the snow-pack is not in contact with the bed. If $A=0$, expression (3) reduces to the theory of McClung (1981) for which a continuous, infinitesimal thin water film was assumed all along the interface. McClung and Clarke (1987) provided estimates of $D^{\mathrm{A}}(x, y)$ for $A \neq 0$. If $A \rightarrow \infty$, all the interface-roughness obstacles are drowned and $\tau_{0} \rightarrow 0$ (an unstable condition). In practice, $D^{*}$ must be either measured or calculated theoretically for the interface in question (see McClung, 1975). For cases in which there is no glide, $D^{*}=D^{A}=0$.

\section{PLANE-STRAIN SOLUTIONS}

In this paper we compare field data from western Norway (1976-present) with predictions at the centre of a long retaining wall (avalanche defence structure) erected perpendicular to the snow-earth interface on a long slope without curvature (Fig. 1). The boundary condition at the top of the snow-pack is taken as a free surface.

The plane-strain configuration is simple enough that one-dimensional analytical solutions are available (e.g. McClung, 1982; McClung and others, 1984) to describe the average pressure on the face of the structure using Equations (1) and (2). These solutions depend only on $v$,

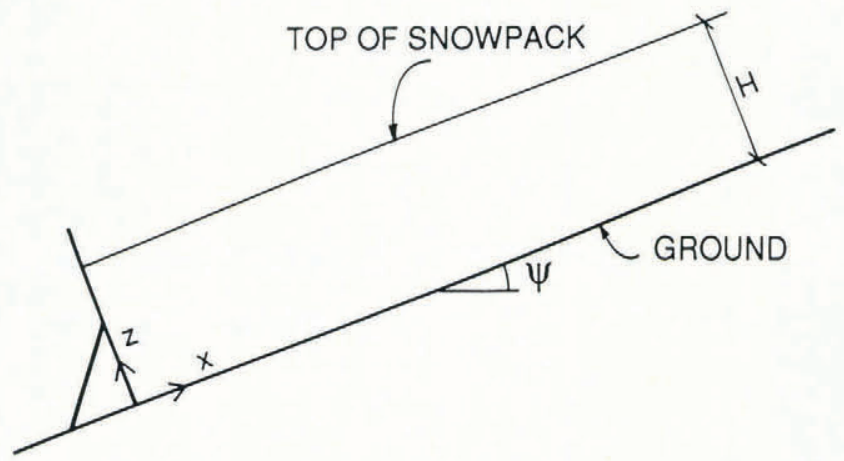

Fig. 1. Schematic of plane-strain configuration for measuring creep pressures.

the slope angle, $\psi$, for a snow-pack with depth-averaged density, $\bar{\rho}$, and constant thickness, $H$.

One-dimensional equations (geometry in Figure 1), are defined by depth-averaged quantities (denoted by a bar):

$$
\bar{\sigma}_{x}=\frac{1}{H} \int_{0}^{H} \sigma_{x x} \mathrm{~d} z
$$

With $D^{*}$ and $v$ taken as constant throughout the zone of influence of the structure, the solution for the compressive stress perpendicular to the structure is given by (McClung, 1982; McClung and Larsen, in press):

$\bar{\sigma}_{x}(0)=\left[\left(\frac{2}{1-v}\right)\left[\frac{D^{*}}{H}+\frac{L}{H}\right)\right]^{\frac{1}{2}} \sin \psi \bar{\rho} g H+\left(\frac{v}{1-v}\right) \bar{\sigma}_{z}(0)$

where $\bar{\sigma}_{z}(0)$ is depth-averaged normal stress at the structure, and $g$ is acceleration due to gravity. In Equation (5), both $\bar{\sigma}_{z}(0)$ and the dimensionless parameter $L / H$ (defined by McClung (1982)) depend on $v, \psi$, and the boundary conditions on the structure.

\section{BOUNDARY CONDITIONS ON THE STRUCTURE: PLANE-STRAIN SOLUTIONS}

The boundary conditions at the face of structures buried in snow covers are unknown. However, it is possible to place bounds on them. Regardless of the conditions of traction or displacement parallel to the structure, the creep velocity perpendicular to that structure may be taken as $u=0$ along the face.

For a rough structure in a cold snow-pack, the vertical creep velocity may be approximated as $v=0$. The pair of boundary creep velocities $(u=v=0$, will be referred to as the no-slip condition. This condition is to be expected from results on snow gliding; glide is not observed on a rough surface unless free water (wet snow) is available. The no-slip condition implies a shear stress along the structure face and causes the maximum force to occur at an angle (rather than perpendicular) to the structure.

At the other extreme, for a smooth structure lubricated by free water, a traction-free condition $\tau_{x z}=0$ along the structure is expected. This pair of conditions $\left(u=\tau_{x z}=0\right)$ is called the traction-free condition. For the intermediate case (both slip and traction occur parallel to the structure), a relation analogous to that in Equation (5) may be appropriate. This situation is not explored here explicitly; it should produce pressures intermediate between the no-slip and traction-free conditions which bound the problem.

In order to make a comparison with field data, it is of interest to predict the forces perpendicular and parallel to the structure (shear forces) as well as the maximum principal stress.

\section{(i) Traction-free boundary condition}

The traction-free boundary condition is the easier of the two extremes to model in one dimension. Also, twodimensional finite-element solutions show that the normal forces are not appreciably changed from their values 
without the presence of the wall, and therefore the depth-averaged normal stress is approximately

$$
\bar{\sigma}_{z}(0) \simeq \frac{1}{2} \bar{\rho} g H \cos \boldsymbol{\psi} .
$$

An extensive series of finite-element solutions (McClung and Larsen, in press) showed that an empirical expression for $L / H$ (traction-free condition) is

$$
\frac{L}{H}=0.27+\frac{v}{12}
$$

With Equations (5)-(7), the depth-averaged value of $\bar{\sigma}_{x}(0)$ (or maximum principal stress, $\bar{\sigma}_{\mathrm{I}}$ ) is given by:

$$
\frac{\bar{\sigma}_{x}(0)}{\bar{\rho} g H}=\left[\left(\frac{2}{1-v}\right)\left[\frac{D^{*}}{H}+\frac{L}{H}\right)\right]^{\frac{1}{2}} \sin \psi+\frac{1}{2}\left[\frac{v}{1-v}\right] \cos \psi .
$$

The two terms in Equation (8) result from gravity loads applied parallel and perpendicular to the slope, respectively. These terms may be calculated separately by application of gravity loads (body forces) in these directions. Equation (8) was derived from solutions in the ranges $25^{\circ} \leqslant \psi \leqslant 55^{\circ}$; $0 \leqslant v \leqslant 0.4$; and $0 \leqslant D^{*} / H \leqslant 3$ (see McClung (1982) for an explanation of these ranges). Maximum stresses determined by using Equation (8) compare with twodimensional finite-element solutions within a few per cent (Figure 2 gives examples). For the traction-free condition, the resultant force is perpendicular to the face of the structure with a magnitude given by Equation (8).

\section{(ii) No-slip boundary condition}

Field data show that, in general, the resultant force is not perpendicular to the face of the structure (e.g. Kümmerli, 1958). This result is expected physically; if slip along the structure is inhibited, shear force will be present causing the resultant force to have a component perpendicular to the slope. In general, the face of a structure will not be completely traction-free, and we feel that the no-slip boundary condition $(u=v=0)$ is a close approximation to conditions encountered in the field except when the snow-pack is melting rapidly.

Numerical solutions show that the no-slip condition is more complex than the traction-free condition to model. Not only are shear forces produced on the structure but the normal forces in the vicinity of the structure are significantly reduced from their values when a structure is not present; a simple estimate such as approximation (6) is not available.

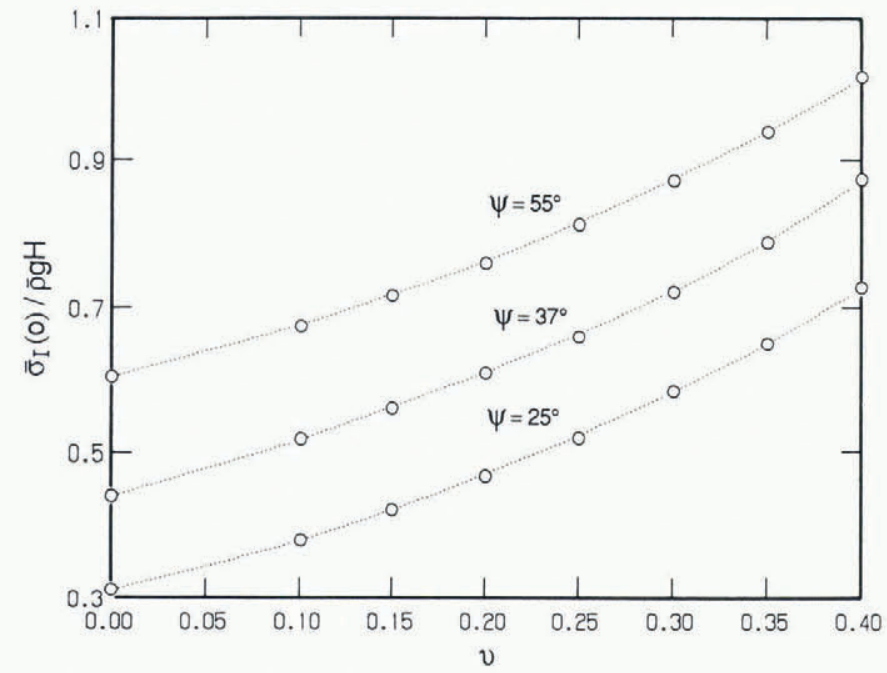

Fig. 2. Comparison of maximum principal stress as a function of the constant-density (depth-averaged)stiffness model for the traction-free boundary condition $D^{*}=0 . \quad(\ldots)$ Model predictions; (0) finite-element calculations.
We have evaluated Equation (5) numerically using finiteelement solutions to get an approximate empirical expression for $\bar{\sigma}_{x}(0)$. If the form of Equation (8) is retained (McClung and Larsen, in press), $L / H$ is given by:

$$
\frac{L}{H} \simeq \frac{1}{4}[\sin \psi]^{\frac{1}{2}}+\frac{1}{4}\left[\frac{v^{2}}{1-v}\right]
$$

which completes the definition of $\bar{\sigma}_{x}(0)$ in Equation (8).

An approximate expression for $\bar{\tau}(0)$ (depth-averaged shear stress on the structure) has also been derived from finite-element calculations:

$$
\frac{\bar{\tau}(0)}{\bar{\rho} g H} \simeq \frac{1}{4}\{\cos \psi]^{\frac{1}{2}}\left[1-\frac{3}{2}\left[\frac{v}{1-v}\right](\sin \psi]^{\frac{1}{2}}\right] .
$$

A note of caution applies to approximations (9) and (10); they are not as accurate as Equation (7) for the traction-free boundary condition and therefore finiteelement solutions are preferable. Errors of up to $15 \%$ may be expected using approximation (10) but the errors are less than $10 \%$ for total resultant force on the structure.

For the no-slip boundary condition, the vertical stress is derived from plane-strain solutions with $\dot{\mathrm{e}}_{z z}=0$ :

$$
\bar{\sigma}_{z}(0)=\frac{v}{1-v} \bar{\sigma}_{x}(0)
$$

to complete the definition of stresses in expressions (8)-(11). The maximum principal stress $\left(\bar{\sigma}_{\mathrm{I}}\right)$ is not equal to $\bar{\sigma}_{x}(0)$ for the no-slip boundary condition but it can be calculated by standard methods using expressions (8), (9), (10), and (11). Figure 3 gives an example.

\section{RESULTANT FORCE AND DIRECTION}

When a structure is erected perpendicular to a slope, the stress components of interest are $\bar{\sigma}_{x}(0)$ and $\bar{\tau}(0)$. The magnitude of the resultant force (per unit area) is

$$
\sigma_{\mathrm{R}}=\left[\bar{\sigma}_{x}(0)^{2}+\tau(0)^{2}\right]^{\frac{1}{2}}
$$

and its direction may be defined (Haefeli, 1948)

$$
\tan \varepsilon=\frac{\bar{\tau}(0)}{\bar{\sigma}_{x}(0)} .
$$

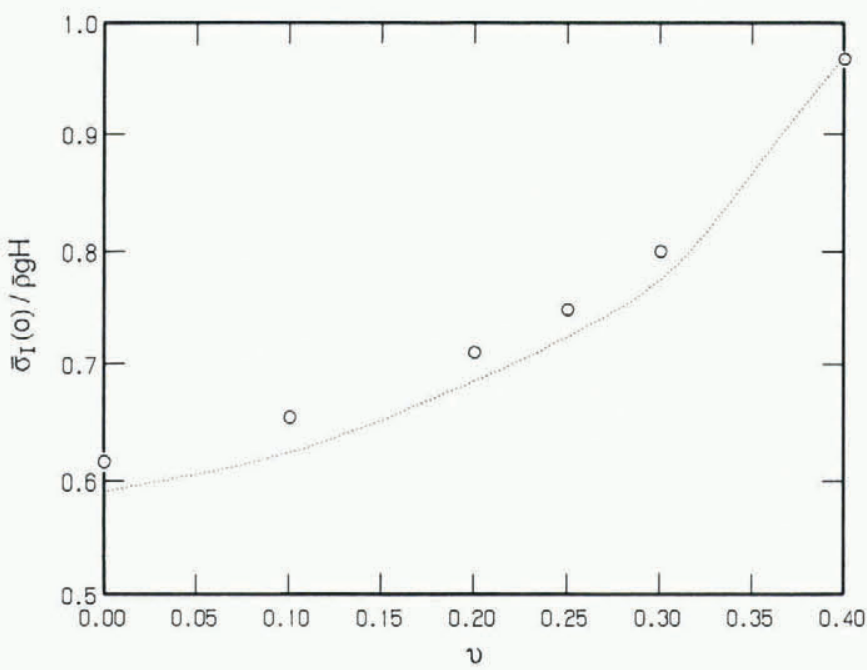

Fig. 3. Model comparison for the same conditions as in Figure 2 for the no-slip boundary condition $\psi=45^{\circ}$. 
The ratio in Equation (13) has been estimated in the field (Kümmerli, 1958). Salm (1977) has given a range for $\tan \varepsilon$ from field measurements. With $\psi=37^{\circ}, D^{*}=0, \tan \varepsilon$ $=0.7$ for low-density snow and $\tan \varepsilon=0.3$ for high-density snow. From Bader's data (Salm, 1977), with $v=0.0$ (low-density snow) and $v=0.25$ (high-density snow), calculations using expressions (5), (9), and (10) give $\tan \varepsilon=$ 0.61 and $\tan \varepsilon=0.29$, respectively. For these same assumptions, finite-element calculations give $\tan \varepsilon=0.77$ $(v=0.0)$ and $\tan \varepsilon=0.36 \quad(v=0.25)$. The agreement is surprisingly good for the linear, depth-averaged density case.

\section{COMPARISON WITH FIELD DATA}

Our data were taken from a low-altitude high-latitude site in western Norway. The region is classed as a maritime climate regime and strong wind-packing effects are present. The experimental procedures have been given by Larsen and others (1985). The pressure data consist of maximum $\left(\sigma_{\mathrm{m}}\right)$ and average pressure $\left(\sigma_{\mathrm{R}}\right)$, estimated at the centre of a $15 \mathrm{~m}$ long avalanche-defence structure erected perpendicular to the slope. The incline at the site is almost constant $\left(25^{\circ}\right)$ for a long distance up-slope. Although the ground surface up-slope from the structure is fairly smooth rock up-slope from the structure, measurements have shown that glide is negligible $\left(D^{*}=0\right)$. We also measured snow-pack properties near the site, including $\bar{\rho}$ and $H$.

Since most of our data were taken when the snow-pack temperature was below $0^{\circ} \mathrm{C}$ (mid-winter), we believe that the no-slip boundary condition is physically more realistic than the traction-free condition. Similar data from Switzerland (Salm, 1977) show clearly that the resultant force is not perpendicular to the structure, indicating the presence of shear forces and very little slip on the face of the structure.

A regression analysis of the field data gives

$$
\begin{aligned}
\bar{\sigma}_{\mathrm{R}} & =0.65 \rho g H \\
r^{2} & =0.70, S_{\mathrm{e}}=1.61 \mathrm{kPa}, \quad N=53
\end{aligned}
$$

where $r$ is the correlation coefficient, $S_{\mathrm{e}}$ is the standard error, and $N$ is the number of data points.

To compare with the model, consider first the average pressure $\sigma_{\mathrm{n}}$ on the face of the structure. For either boundary condition, the ratio $\sigma_{\mathrm{R}} / \bar{\rho} g H$ should depend only on $v$ and $\psi$. From data reviewed by Salm (1977), we regard the extreme range of $v$ as $0-0.4$ for depth-averaged densities varying between 200 and $600 \mathrm{~kg} / \mathrm{m}^{3}$. Finite-

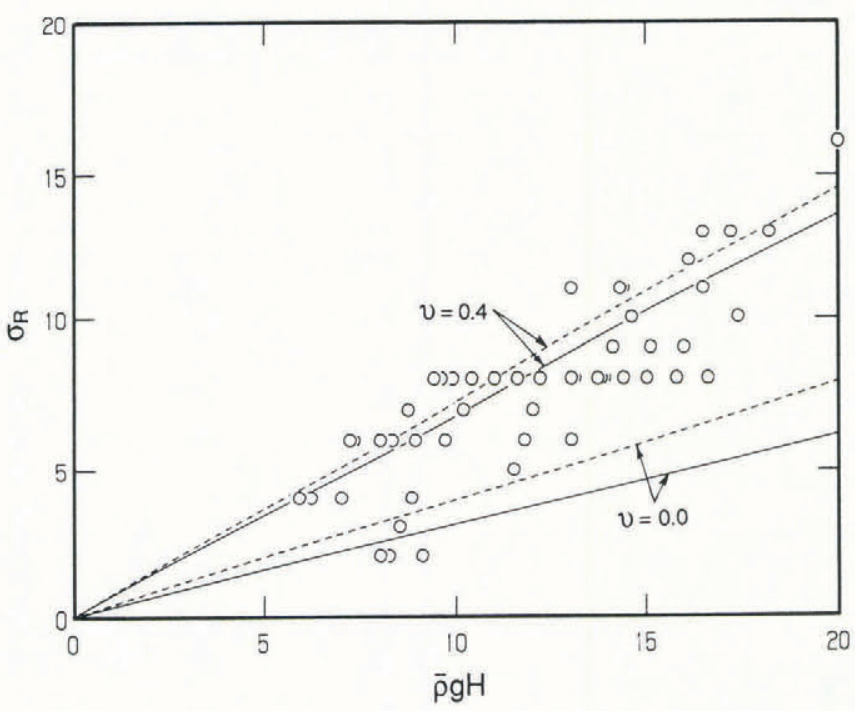

Fig. 4. Comparison of $\sigma_{\mathrm{R}}$ versus $\bar{\rho} g H$ (both in $\mathrm{kPa}$ ) for measurements (o) and finite-element solutions for the ranges of expected structure boundary conditions and $v$. $(\longrightarrow)$, no slip; (...), traction-free. Constant-density (depth-averaged) and stiffness are assumed and $D^{*}=0$. element calculations show that the mean value of our data $\left(\sigma_{\mathrm{R}} / \bar{\rho} g H=0.64\right)$ implies $v \approx 0.4$ and a range of $0-0.45$ for the no-slip boundary condition. From Equations (14), the ratio $\sigma_{\mathrm{R}} / \bar{\rho} g H$ is near 0.65 by regression analysis. For the traction-free boundary condition, the ratio $\sigma_{\mathrm{R}} / \bar{\rho} g H=0.75$ for $v=0.4$.

Figure 4 shows a comparison of the data with calculations (units are in $\mathrm{kPa}$ ) for both boundary conditions for the range $0 \leqslant v \leqslant 0.4$. These comparisons show that $v=0.0$ does provide a good lower bound on our data, but many of the data points lie outside the extreme limits predicted for either boundary condition. It appears that the mean of our data is close to the extreme limits predicted by the linear constant-stiffness model. Bader and others (1951) presented data indicating that $v$ increases with density from 0.1 to 0.25 for the density range in our experiments. Since we have used a wider range of $v$ in our comparison, the underestimates of the model may be even greater than indicated in Figure 4. If Bader's data are accepted as correct, the linear constant-stiffness model underestimates the mean pressures by at least $20 \%$.

Numerical calculations were performed to explore depthdependent density variations. Assuming a linear increase in density with depth, these results showed almost no effect on the average pressure and the maximum pressure changes only slightly for either the no-slip or traction-free boundary condition.

Sensitivity with respect to variations in snow-pack stiffness may be analyzed using viscosity data summarized by Haefeli (1967). He showed that the shear viscosity of snow varies by approximately two orders of magnitude $\left(10^{10}-10^{12} \mathrm{~kg} / \mathrm{m} \mathrm{s}\right)$ for densities in the range $300-500 \mathrm{~kg} / \mathrm{m}^{3}$. We also performed finite-element calculations with both density and stiffness increasing linearly with depth for these ranges. The results show (McClung and Larsen, in press) that the predicted pressures are lower than for the constant-stiffness model for either the traction-free or no-slip boundary condition. For $\psi=25^{\circ}$, the ratio $\sigma_{\mathrm{R}} / \bar{\rho} g H=0.53$ for the no-slip boundary condition with linear variation in stiffness and density, and $v=0.40$. This ratio may be compared with $\sigma_{\mathrm{p}} / \bar{\rho} g H=0.68$ for constant stiffness and the values 0.64 (from the mean of our data) and 0.65 (from the regression analysis).

Our data also contain important information about maximum pressure on the structure. A regression analysis gives

$$
\begin{aligned}
\sigma_{\mathrm{m}} & =1.48 \sigma_{\mathrm{R}}, \\
r^{2} & =0.91, S_{\mathrm{e}}=1.36 \mathrm{kPa}, \quad N=55 .
\end{aligned}
$$

For the constant density-viscosity model, the calculated ratio $\left(\sigma_{\mathrm{m}} / \sigma_{\mathrm{R}}\right)$ declines from 1.39 to 1.18 as $v$ increases from 0 to 0.4 (no-slip boundary condition). From finite-element calculations, $\sigma_{\mathrm{m}} / \sigma_{\mathrm{R}}$ decreases from 1.54 to 1.46 for the same range of $v$, if both density and stiffness increase linearly with depth, thereby providing an excellent fit to our data. For the less-realistic traction-free boundary condition, the ratio decreases from 1.43 to 1.11 as $v$ increases from 0 to 0.4 .

\section{SUMMARY AND DISCUSSION}

Our re-formulation of the constant viscosity-constant density one-dimensional treatment of the plane-strain snowpressure problem is of both historical and practical interest. The solution represents the analytical model first sought by Haefeli (Bader and others, 1939) in his doctoral thesis. Also, our analytical model is of practical interest, since it allows average pressures to be roughly estimated using a hand-calculator. It appears that this simple model underestimates our field data by about $20 \%$.

The analytical model we have presented departs from previous formulations, because initial stresses are not accounted for. Instead, the long-term loads on the structure are defined in terms of viscous stresses and strain-rates. These assumptions have a long history in snow mechanics (e.g. Mellor, 1975). We feel that the new model presents a more accurate representation of the linear problem than our 
previous attempts which included initial stresses.

Comparison of field data with the two models (constant and linearly varying stiffness and density) shows that neither can explain the high values of average pressure or the width of the data-scatter band. Since density variations alone do not provide a consistent match to field data, we believe that variations in stiffness (non-linear viscous relations) are the key. Our attempt to vary the stiffness (linear increase with depth) is the simplest approach consistent with snow-deformation properties in the field (McClung, 1975). It is encouraging that linear variations in stiffness appear to match the ratio $\sigma_{\mathrm{m}} / \sigma_{\mathrm{p}}$ for our data. Calculations assuming linear variation in stiffness with depth (McClung and Larsen, in press) have shown that most of the data are far in excess of the limits implied by the expected upper range of $v(0.4)$. It is tempting to attribute the data which fall outside the limits in Figure 4 to data scatter. However, the assumption of constant stiffness (Fig. 4) will not allow an explanation of the high values of $\sigma_{\mathrm{m}} / \sigma_{\mathrm{R}}$. We believe that a comprehensive explanation of the field data will require a non-linear viscous relation. Until a non-linear deformation law is formulated and applied, a proper treatment of stiffness variations will not be possible. Based on finite-element solutions and our data, we recommend a safety factor of at least $25 \%$ over the analytical model (constant stiffness) when expected values for $v$ (e.g. Bader and others, 1951) are applied. For maximum pressure, our data indicate that values should be considered to be $50 \%$ higher than predicted average pressures.

\section{ACKNOWLEDGEMENTS}

We are grateful to K. Kristensen for assistance with the field measurements. We also wish to thank the Norwegian Water Power and Electricity Board for funding part of the project.

\section{REFERENCES}

Bader, H., and 6 others. 1939. Der Schnee und seine Metamorphose. Beit. Schweiz. Geotech. Ser. Hydrol., 3 [English translation: SIPRE Translation 14, 1954.]

Bader, H., B.L. Hansen, J.H. Joseph, and M.A. Sandgren. 1951. Preliminary investigations of some physical properties of snow. SIPRE Rep. 7.
Flügge, W. 1975. Viscoelasticity. New York, SpringerVerlag.

Haefeli, R. 1967. Some mechanical aspects on the formation of avalanches. In Ōura, H., ed. Physics of Snow and Ice. International Conference on Low Temperature Science ... 1966 ... Proceedings, Vol. 1. Pt. 2. Sapporo, Institute of Low Temperature Science, 1199-1213.

Kümmerli, F. Unpublished. Auswertung der Druckmessungen am Druckapparat Institut (DAI). Eidgenössisches Institut für Schnee- und Lawinenforschung, Interior Berricht 240, 1958.

Lang, T.E. and T. Nakamura. 1984. Finite element computer analysis of snow settlement. Res. Notes Natil. Res. Cent. Disaster Prevention 59.

Larsen, J.O., D.M. McClung, and S.B. Hansen. 1985. The temporal and spatial variation of snow pressure on structures. Can. Geotech. J., 22(2), 166-171.

McClung, D.M. 1975. Creep and the snow-earth interface condition in the seasonal alpine snowpack. International Association of Hydrological Sciences Publication 114 (Symposium at Grindelwald 1974 - Snow Mechanics). 236-248.

McClung, D.M. 1981. A physical theory of snow gliding. Can. Geotech. J., 18(1), 86-95.

McClung, D.M. 1982. A one-dimensional analytical model for snow creep pressures on rigid structures. Can. Geotech. J., 19(4), 401-412.

McClung, D.M. and G.K.C. Clarke. 1987. The effects of free water on snow gliding. J. Geophys. Res., 92(B7), 6301-6309.

McClung, D.M. and J.O. Larsen. In press. Snow creep pressures: effects of structure boundary conditions and snowpack properties compared with field data. Cold Reg. Sci. Technol.

McClung, D.M., J.O. Larsen, and S.B. Hansen. 1984 Comparison of snow pressure measurements and theoretical predictions. Can. Geolech. J., 21(2), 250-258.

Reiner, M. 1946. The coefficient of viscous traction. Am J. Math., 68, 672-680.

Reiner, M. 1949. On volume - or isotropic flow as exemplified in the creep of concrete. Appl. Sci. Res., Ser. $A, 1,475-488$

Salm, B. 1967. An attempt to clarify triaxial creep mechanics of snow. In Oura, H., ed. Physics of Snow and Ice. International Conference on Low Temperature Science ... 1966 ... Proceedings, Vol. 1, Pt. 2. Sapporo, Institute of Low Temperature Science, 1199-1213.

Salm, B. 1977. Snow forces. J. Glaciol., 19(81), 67-100. 